\title{
Fixed points and adjacent ascents for classical complex reflection groups ${ }^{1}$
}

\author{
Francesco Brenti \\ Dipartimento di Matematica \\ Università di Roma "Tor Vergata" \\ Via della Ricerca Scientifica, 1 \\ 00133 Roma, Italy \\ brenti@mat.uniroma2.it \\ Mario Marietti \\ Dipartimento di Ingegneria Industriale e Scienze Matematiche \\ Università Politecnica delle Marche \\ Via Brecce Bianche \\ 60131 Ancona, Italy \\ m.marietti@univpm.it
}

\begin{abstract}
We characterize the classical complex reflection groups for which a recent symmetric group equidistribution result studied by Diaconis, Evans, and Graham holds. This leads to some refinements of the original result, which seem to be new even in the symmetric group case.
\end{abstract}

\section{Introduction}

In a recent paper [5] Diaconis, Evans, and Graham give various proofs of the fact that the number of permutations in $S_{n}$ with a given set $I$ of fixed points distinct from $n$ is equal to the number of permutations in $S_{n}$ having $I$ as a set of unseparated pairs, for all $I \subseteq[n-1]$ (see $\S 2$ Theorem 2.2 for the relevant definitions).

This result is equivalent to the fact that the two sets of permutations $A=\{\sigma \in$ $\left.S_{n}:\{k \in[n-1]: \sigma(k+1)=\sigma(k)+1\} \supseteq I\right\}$ and $B=\left\{\sigma \in S_{n}:\{k \in[n-1]:\right.$

\footnotetext{
${ }^{1} 2010$ Mathematics Subject Classification: Primary 05A05; Secondary 05E15, 20 F55.
} 
$\sigma(k)=k\} \supseteq I\}$ have the same cardinality for all $I \subseteq[n-1]$. In this paper, we first show that these two sets are just the two "extreme cases" of a large class of subsets of $S_{n}$, all having the same cardinality: these subsets of permutations are indexed by a pair $(J, K)$ of subsets of $[n-1]$ and have cardinalities that depend only on $|J|+|K|$. The two subsets $A$ and $B$ are obtained as special cases with the indexing pairs $(\emptyset, I)$ and $(I, \emptyset)$. We believe that this result sheds some new light on Theorem 2.2. We then investigate the extent to which Theorem 2.2 continues to hold for the classical complex reflection groups, which are the colored permutation groups $G(r, p, n)$, where $r, p, n \in \mathbb{P}$ and $p \mid r$ (see $\S 2$ for the definition). More precisely, we show that, with suitable definition of "unseparated pair "(which we prefer to call "adjacent ascent"), the result holds for the group $G(r, p, n)$ if and only if $\operatorname{gcd}(p, n)=$ 1 (Corollary 4.2). Our investigation naturally leads to a refinement of the result in [5] (Corollary 4.8), which, in turn, holds for $G(r, p, n)$ if and only if all divisors of $p$ are larger than $n$ (Corollary 4.3). We also investigate some other possible definitions of adjacent ascent for colored permutations and the corresponding equidistribution results. Our proofs are enumerative and combinatorial.

The organization of the paper is as follows. In the next section, we recall some definitions, notation, and results that are used in what follows. In $\S 3$, we introduce the concept of compatible subsets and obtain a result that includes the one in [5] as the two "extreme cases". In $\S 4$, we propose a definition of adjacent ascent for any colored permutation and characterize the groups $G(r, p, n)$ for which the result in [5] holds. This naturally leads to a refinement of the original theorem, and we characterize the groups $G(r, p, n)$ for which this refinement holds. This refinement seems to be new even in the symmetric group case. In $\S 5$, we investigate two other possible definitions of "adjacent ascent" for colored permutations and the corresponding equidistribution results. In $\S 6$, we provide bijective proofs of two of the results in the previous sections.

\section{Preliminaries}

In this section we recall some notation, definitions, and results that are used in what follows.

We let $\mathbb{Z}, \mathbb{P}$, and $\mathbb{N}$ be the set of integers, positive integers, and nonnegative integers, respectively. Given $n, m \in \mathbb{N}$, with $n \leq m$, we let $[n, m]:=\{n, n+1, \ldots, m\}$ 
and, for $n \in \mathbb{P}$, we let $[n]:=[1, n]$. For a set $T$, we let $S(T)$ be the set of all bijections of $T$, and $S_{n}:=S([n])$.

Let $n \in \mathbb{P}$. By a composition of $n$ we mean a sequence $\alpha=\left(\alpha_{1}, \ldots, \alpha_{s}\right)$ (for some $s \in \mathbb{P}$ ) of positive integers such that $\sum_{i=1}^{s} \alpha_{i}=n$. We let $\mathcal{C}_{n}$ be the set of all compositions of $n$, and $\mathcal{C}:=\bigcup_{n \geq 1} \mathcal{C}_{n}$. Given $\beta, \alpha \in \mathcal{C}_{n}$, with $\beta=\left(\beta_{1}, \ldots, \beta_{s}\right)$ and $\alpha=\left(\alpha_{1}, \ldots, \alpha_{t}\right)$, we say that $\beta$ refines $\alpha$ if there exist $1 \leq i_{1}<i_{2}<\cdots<i_{t-1}<s$ such that $\sum_{j=i_{k-1}+1}^{i_{k}} \beta_{j}=\alpha_{k}$ for $k=1, \ldots, t$ (where $i_{0}:=0, i_{t}:=s$ ). We then write $\beta \preceq \alpha$. Moreover, for $\beta=\left(\beta_{1}, \ldots, \beta_{s}\right) \in \mathcal{C}_{n}$, we let $\pi(\beta)$ be the non-increasing rearrangement of $\beta_{1}, \ldots, \beta_{s}$. For any other undefined notation and terminology in enumerative combinatorics, we follow [10] and [11].

Let $r, n \in \mathbb{P}$. We denote by $G(r, n)$ the group of colored permutations of $[n]$ with $r$ colors. So the underlying set is $[0, r-1]^{n} \times S_{n}$, and, for $\left(r_{1}, \ldots, r_{n}, \sigma\right),\left(s_{1}, \ldots, s_{n}, \tau\right) \in$ $G(r, n)$, we have

$$
\left(r_{1}, \ldots, r_{n}, \sigma\right)\left(s_{1}, \ldots, s_{n}, \tau\right)=\left(r_{\tau(1)}+s_{1}, \ldots, r_{\tau(n)}+s_{n}, \sigma \tau\right)
$$

where the sums are taken modulo $r$. Given $p \in \mathbb{P}$, with $p \mid r$, we let

$$
G(r, p, n):=\left\{\left(r_{1}, \ldots, r_{n}, \sigma\right) \in G(r, n): p \mid\left(r_{1}+\cdots+r_{n}\right)\right\}
$$

It is clear that $G(r, p, n)$ is a subgroup of $G(r, n)$ of index $p$.

Let $\xi \in \mathbb{C}$ be a primitive $r$-th root of unity. We identify $G(r, n)$ with the group of all $n \times n$ complex matrices with entries in $\left\{\xi^{k}: k \in[r]\right\} \cup\{0\}$ that have exactly one nonzero entry in each row and column by identifying $\left(r_{1}, \ldots, r_{n}, \sigma\right)$ with the matrix $M \in M_{n}(\mathbb{C})$ defined by

$$
M_{i, j}:= \begin{cases}\xi^{r_{j}}, & \text { if } i=\sigma(j), \\ 0, & \text { otherwise }\end{cases}
$$

for all $i, j \in[n]$. We also identify $G(r, n)$ with the group of all bijections $f \in$ $S([0, r-1] \times[n])$ such that $f(t, i)=(t, 0)+f(0, i)$ for all $(t, i) \in[0, r-1] \times[n]$, where the sum in the first component is taken modulo $r$, by identifying $\left(r_{1}, \ldots, r_{n}, \sigma\right)$ with the function $g:[0, r-1] \times[n] \rightarrow[0, r-1] \times[n]$ defined by $g(t, i):=\left(t+r_{i}, \sigma(i)\right)$ for all $(t, i) \in[0, r-1] \times[n]$. It is clear that any such bijection $f \in S([0, r-1] \times[n])$ is uniquely determined by its values on $\{0\} \times[n]$, and we write

$$
f=\left[a_{1}^{c_{1}}, \ldots, a_{n}^{c_{n}}\right]
$$


to mean that $f(0, i)=\left(c_{i}, a_{i}\right)$, for all $i \in[n]$. Note that $\left(r_{1}, \ldots, r_{n}, \sigma\right)$ corresponds to $\left[\sigma(1)^{r_{1}}, \ldots, \sigma(n)^{r_{n}}\right]$. We call (1) the window notation of $f$. We sometimes find it convenient to write $a^{c}$ for $(c, a)$. Following [4] (see also [1], [2], and the references cited there), we let

$$
F(g):=\left\{i \in[n]:\left(\sigma(i), r_{i}\right)=(i, 0)\right\} .
$$

for $g=\left(r_{1}, \ldots, r_{n}, \sigma\right) \in G(r, n)$. We call the elements of $F(g)$ the fixed points of $g$. In what follows, we use the convention that $\sigma(0)=0, \sigma(n+1)=n+1$, and $r_{0}=r_{n+1}=0$, for all $\left(r_{1}, \ldots, r_{n}, \sigma\right) \in G(r, n)$.

It is well known that the groups $G(r, p, n)$ (and so, in particular, the groups $G(r, n)=G(r, 1, n))$ are all complex reflection groups. They are usually referred to as the classical complex reflection groups. Moreover, the groups $G(1,1, n), G(2,1, n)$, and $G(2,2, n)$ are isomorphic to the Coxeter groups of types $A_{n-1}, B_{n}$, and $D_{n}$, respectively. When working with these Coxeter groups, we prefer to use their usual presentations as groups of permutations (see e.g. [3, Chapter 8]). So we let $B_{n}$ be the group of all permutations $\sigma$ of the set $-[n] \cup[n]$ such that $\sigma(-i)=-\sigma(i)$, for all $i \in-[n] \cup[n]$. Because of this presentation, $B_{n}$ is often called the "signed permutation group". Similarly, we let $D_{n}$ be the subgroup of $B_{n}$ consisting of the signed permutations $\tau$ such that $|\{i \in[n]: \tau(i)<0\}| \equiv 0(\bmod 2)$, which is often called the "even-signed permutation group".

The following result is well known (see, for example, [13, Theorems 3.2.3 and $3.2 .4])$.

Proposition 2.1. Let $a_{1}, \ldots, a_{h}, b \in \mathbb{Z}$, and $m \in \mathbb{P}$. There exist $\left[x_{1}\right]_{m}, \ldots,\left[x_{h}\right]_{m} \in$ $\mathbb{Z}_{m}$ such that

$$
\left[a_{1}\right]_{m}\left[x_{1}\right]_{m}+\cdots+\left[a_{h}\right]_{m}\left[x_{h}\right]_{m}=[b]_{m}
$$

if and only if $\operatorname{gcd}\left(a_{1}, \ldots, a_{h}, m\right) \mid b$.

In this case, the number of h-tuples $\left(\left[x_{1}\right]_{m}, \ldots,\left[x_{h}\right]_{m}\right) \in\left(\mathbb{Z}_{m}\right)^{h}$ that are solutions of (2) is equal to $m^{h-1} \cdot \operatorname{gcd}\left(a_{1}, \ldots, a_{h}, m\right)$.

The following result was proved in [5] (see Theorem 1.1 and the comments following it).

Theorem 2.2. Let $n \in \mathbb{P}$. For all $I \subseteq[n-1]$,

$$
\left|\left\{\sigma \in S_{n}:\{k \in[n-1]: \sigma(k+1)=\sigma(k)+1\}=I\right\}\right|
$$




$$
=\left|\left\{\sigma \in S_{n}:\{k \in[n-1]: \sigma(k)=k\}=I\right\}\right| .
$$

Diaconis, Evans, and Graham call a pair $(k, k+1)$, with $k \in[n-1]$, an "unseparated pair" of the permutation $\sigma$ provided that $\sigma(k+1)=\sigma(k)+1$. The name, and the motivation for studying this concept, comes from thinking of a permutation as the result of shuffling a deck of $n$ cards. Thus, an unseparated pair is a pair of cards that are adjacent at the beginning of the shuffle and are still adjacent at the end of it. This concept has also been studied in the literature under the name of "succession" (see, e.g., [7, 8, 9, 12], and the references cited there).

Our aim in this paper is to investigate the extent to which Theorem 2.2 continues to hold for the classical complex reflection groups $G(r, p, n)$.

\section{Compatible subsets}

In [5], Diaconis, Evans, and Graham give three different proofs of Theorem 2.2. The first proof that they give is enumerative and follows directly by the Principle of Inclusion-Exclusion from the fact that, for all $I \subseteq[n-1]$, the sets $\left\{\sigma \in S_{n}: \sigma(i)=\right.$ $i, \forall i \in I\}$ and $\left\{\sigma \in S_{n}: \sigma(i+1)=\sigma(i)+1, \forall i \in I\right\}$ have both cardinality $(n-|I|) !$. In this section, we show that this is a special case of a more general phenomenon.

Definition 3.1. For any subset $J \subseteq[n-1]$, we let $P_{J}$ be the interval partition of $[n]$ whose blocks are the intervals $[t, j]$ such that $[t, j-1] \subseteq J$ and $t-1, j \notin J$.

Note that $[t, j-1]$ could be empty, and that each block of $P_{J}$ contains exactly one element not in $J$, so that $P_{J}$ has $n-|J|$ blocks.

Fix $J \subseteq[n-1]$. Given $I \subseteq[n] \backslash J$, we say that a block $B \in P_{J}$ is

- $I$-free, if $B \cap I=\emptyset$;

- $I$-fixed, otherwise.

Note that, if the block $B$ is fixed, then $B \cap I$ is the singleton consisting of the greatest element of the interval $B$, since this element is the only one in $B \backslash J$. Hence the $I$-fixed and $I$-free blocks of $P_{J}$ are, respectively, $|I|$ and $n-|J|-|I|$ in number.

Definition 3.2. We say that a subset $I \subseteq[n]$ is $J$-compatible if $I \cap J=\emptyset$ and either all $I$-free blocks of $P_{J}$ have the same cardinality or their union is an interval. 
For example, let $n=12, J=\{2,5,6,9,11\}$, and $I=\{1,4,7,8\}$. Then

$$
P_{J}=\mathbf{1}|2,3| \mathbf{4}|5,6, \mathbf{7}| \mathbf{8}|9,10| 11,12
$$

has four $I$-fixed blocks (those containing an element in $I$, bolded) and three $I$-free blocks. The subset $I$ is $J$-compatible since the three $I$-free blocks have the same cardinality. On the other hand, also $I^{\prime}=\{1,8,10,12\}$ is $J$-compatible since the partition

$$
P_{J}=\mathbf{1}|2,3| 4|5,6,7| \mathbf{8}|9, \mathbf{1 0}| 11, \mathbf{1 2}
$$

has three $I^{\prime}$-free blocks whose union forms the interval $[2,7]$.

We can now state and prove the main result of this section.

Theorem 3.3. Let $J \subseteq[n-1]$ and $I \subseteq[n]$. If $I$ is $J$-compatible, then

$$
\mid\left\{\sigma \in S_{n}: \sigma(i)=i, \forall i \in I, \text { and } \sigma(j+1)=\sigma(j)+1, \forall j \in J\right\} \mid=(n-|I|-|J|) !
$$

Proof. For short, we set $\mathcal{S}=\mathcal{S}_{I, J}=\left\{\sigma \in S_{n}: \sigma(i)=i, \forall i \in I\right.$, and $\sigma(j+1)=$ $\sigma(j)+1, \forall j \in J\}$.

Given $\tau \in S_{n}$, we have $\tau \in \mathcal{S}^{-1}$ if and only if $\tau(i)=i$, for all $i \in I$, and $j$ and $j+1$ are adjacent in this order in the window notation of $\tau$, for all $j \in J$. Therefore $\tau \in \mathcal{S}^{-1}$ if and only if $\tau(i)=i$, for all $i \in I$, and $t, t+1, \ldots, j$ are adjacent in this order in the window notation of $\tau$ whenever $[t, j]$ is a block of $P_{J}$. Hence $\tau \in \mathcal{S}^{-1}$ is equivalent to the following two conditions:

- $t, t+1, \ldots, j$ are adjacent in this order in the window notation of $\tau$, for every block $[t, j]$ of $P_{J}$

- $t, t+1, \ldots, j$ appear in positions $t, t+1, \ldots, j$, respectively, for every $I$-fixed block $[t, j]$ of $P_{J}$.

Since $I$ is $J$-compatible, all permutations of the $I$-free blocks of $P_{J}$ occur in elements of $\mathcal{S}^{-1}$, so $\left|\mathcal{S}^{-1}\right|=m$ !, where $m$ is the number of $I$-free blocks of $P_{J}$, and the result follows.

In other words, we have just shown that, under the hypotheses of Theorem 3.3, the symmetric group $S_{m}$ acts faithfully and transitively on $\mathcal{S}_{I, J}{ }^{-1}$ by permuting the $I$-free blocks of $P_{J}$ in the window notations (here $m=n-|I|-|J|$ is the number of $I$-free blocks). 
Remark 3.4. For all $I \subseteq[n-1]$, we have that $I$ is $\emptyset$-compatible and $\emptyset$ is $I$-compatible. Indeed,

- all blocks of $P_{\emptyset}$ have cardinality 1 , and so, in particular, all $I$-free blocks of $P_{\emptyset}$ are equinumerous;

- all blocks of $P_{I}$ are $\emptyset$-free, and so their union is the interval $[1, n]$.

Applying Theorem 3.3 in these two special cases, we obtain

$$
\left|\left\{\sigma \in S_{n}: \sigma(i)=i, \forall i \in I\right\}\right|=(n-|I|) !
$$

and

$$
\left|\left\{\sigma \in S_{n}: \sigma(i+1)=\sigma(i)+1, \forall i \in I\right\}\right|=(n-|I|) !
$$

from which Theorem 2.2 follows.

\section{Equidistribution results for the groups $G(r, p, n)$}

In this section, we define the concept of adjacent ascent for any colored permutation and investigate the extent to which the result of Diaconis, Evans, and Graham continues to hold, with this definition, for the groups $G(r, p, n)$. We show that the result holds for $G(r, p, n)$ if and only if $\operatorname{gcd}(p, n)=1$ and that a refinement of it holds if and only if all divisors of $p$ are larger than $n$. This last result seems to be new even in the symmetric group case.

Given $g=\left(s_{1}, \ldots, s_{n}, \sigma\right) \in G(r, n)$, we let

$$
A A(g):=\left\{i \in[n]:\left(\sigma(i+1), s_{i+1}\right)=\left(\sigma(i)+1, s_{i}\right)\right\} .
$$

We call the elements of $A A(g)$ the adjacent ascents of $g$.

Given $I, J \subseteq[n]$, we let

$$
\mathcal{G}_{I, J}(r, p, n):=\{g \in G(r, p, n): F(g) \supseteq I, A A(g) \supseteq J\} .
$$

Note that $n \in F(g)$ if and only if $n \in A A(g)$, for all $g \in G(r, p, n)$ (recall our convention that $\sigma(n+1)=n+1$ and $s_{n+1}=0$, for all $\left.\left(s_{1}, \ldots, s_{n}, \sigma\right) \in G(r, n)\right)$. Hence $\mathcal{G}_{I, J \cup\{n\}}(r, p, n)=\mathcal{G}_{I \cup\{n\}, J}(r, p, n)=\mathcal{G}_{I \cup\{n\}, J \cup\{n\}}(r, p, n)$.

Let $I \subseteq[n]$ and $J \subseteq[n-1]$, with $I \cap J=\emptyset$. Let $B_{1}, \ldots, B_{n-|I|-|J|}$ be the $I$-free blocks of $P_{J}$ and $A_{1}, \ldots, A_{|I|}$ be the $I$-fixed blocks of $P_{J}$. Let $\lambda(I, J)$ be the nonincreasing rearrangement of $\left|B_{1}\right|, \ldots,\left|B_{n-|I|-|J|}\right|$ and $\alpha(I, J):=\left(\alpha_{1}, \ldots, \alpha_{s}\right)$ where 
$\alpha_{i}$ is the size of the $i$-th (from the left, say) connected component of $[n] \backslash\left(\cup_{i=1}^{|I|} A_{i}\right)$. Given a partition $\lambda=\left(\lambda_{1}, \ldots, \lambda_{k}\right)$ we let, as customary, $m_{i}(\lambda)=\left|\left\{j \in[k]: \lambda_{j}=i\right\}\right|$, for all $i \in \mathbb{P}$.

Theorem 4.1. Let $p, r, n \in \mathbb{P}$, with $p \mid r$. If $I \subseteq[n]$ and $J \subseteq[n-1]$, with $I \cap J=\emptyset$, then $\left|\mathcal{G}_{I, J}(r, p, n)\right|$ equals

$$
\operatorname{gcd}\left(\lambda_{1}, \ldots, \lambda_{n-|I|-|J|}, p\right) \cdot \frac{r^{n-|I|-|J|}}{p} \cdot|\{\beta \in \mathcal{C}: \beta \preceq \alpha(I, J), \pi(\beta)=\lambda\}| \cdot \prod_{i \geq 1} m_{i}(\lambda) !
$$

where $\lambda:=\lambda(I, J)=\left(\lambda_{1}, \ldots, \lambda_{n-|I|-|J|}\right)$.

Proof. For short, we set $\mathcal{G}:=\mathcal{G}_{I, J}(r, p, n)$. Let $\left(s_{1}, \ldots, s_{n}, \tau\right) \in G(r, p, n)$. It follows from our definitions that $\left(s_{1}, \ldots, s_{n}, \tau\right) \in \mathcal{G}^{-1}$ if and only if $\tau(i)=i$ and $s_{i}=0$ for all $i \in I$, and $j$ and $j+1$ have the same color and are adjacent in this order in the window notation of $\left(s_{1}, \ldots, s_{n}, \tau\right)$ for all $j \in J$. Therefore $\left(s_{1}, \ldots, s_{n}, \tau\right) \in \mathcal{G}^{-1}$ if and only if $\tau(i)=i$ and $s_{i}=0$ for all $i \in I$, and $t, t+1, \ldots, j$ all have the same color and are adjacent in this order in the window notation of $\left(s_{1}, \ldots, s_{n}, \tau\right)$ whenever $[t, j]$ is a block of $P_{J}$. Hence $\left(s_{1}, \ldots, s_{n}, \tau\right) \in \mathcal{G}^{-1}$ if and only if

- $t, t+1, \ldots, j$ have the same color and are adjacent in this order in the window notation of $\left(s_{1}, \ldots, s_{n}, \tau\right)$, for every block $[t, j]$ of $P_{J}$, and

- furthermore, this common color is 0 , and $t, t+1, \ldots, j$ appear in positions $t, t+1, \ldots, j$, respectively, if $[t, j]$ is a fixed block.

Let $u:=\left(s_{1}, \ldots, s_{n}, \tau\right) \in \mathcal{G}^{-1}$, and let $\beta(u):=\left(\beta_{1}, \ldots, \beta_{m}\right)$ where $m=n-|I|-|J|$ and $\beta_{i}$ is the size of the $i$-th (from the left) free block of $P_{J}$ as it appears in the window notation of $u$. Then $\beta(u) \preceq \alpha(I, J)$ and $\pi(\beta(u))=\lambda$. Furthermore, given $\beta \in \mathcal{C}$ such that $\beta \preceq \alpha(I, J)$ and $\pi(\beta)=\lambda$, there are $\operatorname{gcd}\left(\lambda_{1}, \ldots, \lambda_{m}, p\right) \cdot \frac{r^{m}}{p} \cdot \prod_{i \geq 1} m_{i}(\lambda)$ ! elements $u \in \mathcal{G}^{-1}$ such that $\beta(u)=\beta$. Indeed, for each $i \geq 1$, the $m_{i}(\lambda)$ free blocks of $P_{J}$ of size $i$ can be permuted arbitrarily without changing $\beta(u)$. Furthermore, if $s_{i}$ is the common color of the $i$-th (from the left) free block of $P_{J}$ as above, then we have

$$
\left[s_{1}\right]_{p}\left[\beta_{1}\right]_{p}+\cdots+\left[s_{m}\right]_{p}\left[\beta_{m}\right]_{p}=[0]_{p} .
$$

By Proposition 2.1, the number of $m$-tuples $\left(\left[s_{1}\right]_{p}, \ldots,\left[s_{m}\right]_{p}\right) \in \mathbb{Z}_{p}^{m}$ that satisfy (3) is $p^{m-1} \operatorname{gcd}\left(\beta_{1}, \ldots, \beta_{m}, p\right)$, and so the number of $m$-tuples $\left(s_{1}, \ldots, s_{m}\right) \in[0, r-1]^{m}$ that satisfy $(3)$ is $\left(\frac{r}{p}\right)^{m} p^{m-1} \operatorname{gcd}\left(\beta_{1}, \ldots, \beta_{m}, p\right)$. The result follows. 
As a consequence of Theorem 4.1, we obtain the characterization of the groups $G(r, p, n)$ for which the analogue of Theorem 2.2 holds.

Corollary 4.2. Let $r, p, n \in \mathbb{P}$, with $p \mid r$. The following are equivalent:

- $\operatorname{gcd}(p, n)=1$,

- for all $I \subseteq[n-1]$,

$$
|\{g \in G(r, p, n): F(g) \cap[n-1]=I\}|=|\{g \in G(r, p, n): A A(g) \cap[n-1]=I\}| .
$$

In this case,

$$
\begin{aligned}
\mid\{g & \in G(r, p, n):|F(g) \cap[n-1]|=m\}|=|\{g \in G(r, p, n):|A A(g) \cap[n-1]|=m\} \mid \\
& =\frac{(n-1) !}{p} \sum_{k=m}^{n-1}(-1)^{k-m} r^{n-k} \frac{n-k}{m !(k-m) !}
\end{aligned}
$$

for all $0 \leq m \leq n-1$

Proof. Suppose $\operatorname{gcd}(p, n)=1$. Let $I \subseteq[n-1]$. Applying Theorem 4.1 to $(I, \emptyset)$, we obtain $\lambda(I, \emptyset)=\left(1^{n-|I|}\right)$, and so

$$
\left|\mathcal{G}_{I, \emptyset}(r, p, n)\right|=(n-|I|) ! \frac{r^{n-|I|}}{p}
$$

(indeed we do not use the hypothesis $\operatorname{gcd}(p, n)=1$ here). On the other hand, applying Theorem 4.1 to $(\emptyset, I)$, we obtain $\alpha(\emptyset, I)=(n)$, and so

$$
\begin{aligned}
& \left|\mathcal{G}_{\emptyset, I}(r, p, n)\right|=\operatorname{gcd}\left(\lambda_{1}, \ldots, \lambda_{n-|I|}, p\right) \cdot \frac{r^{n-|I|}}{p}|\{\beta \in \mathcal{C}: \pi(\beta)=\lambda\}| \prod_{i \geq 1} m_{i}(\lambda) ! \\
& =(n-|I|) ! \frac{r^{n-|I|}}{p}
\end{aligned}
$$

since, as is well known, $|\{\beta \in \mathcal{C}: \pi(\beta)=\lambda\}|=\frac{(n-|I|) !}{\prod_{i \geq 1} m_{i}(\lambda) !}$, and $\operatorname{gcd}\left(\lambda_{1}, \ldots, \lambda_{n-|I|}, p\right)=$ 1 because $\lambda_{1}+\cdots+\lambda_{n-|I|}=n$ and $\operatorname{gcd}(p, n)=1$. Applying the Principle of InclusionExclusion over all $I \subseteq[n-1]$ yields the claimed identity.

Conversely, suppose $\operatorname{gcd}(p, n)>1$. For example, if $I=[n-1]$ then

$$
\begin{aligned}
|\{g \in G(r, p, n): F(g) \cap[n-1]=[n-1]\}| & =|\{(0, \ldots, 0, s, I d) \in G(r, p, n)\}| \\
& =\frac{r}{p}
\end{aligned}
$$


while, if $p^{\prime}:=\frac{p}{\operatorname{gcd}(p, n)}$, then

$$
\begin{aligned}
|\{g \in G(r, p, n): A A(g) \cap[n-1]=[n-1]\}| & =|\{(s, \ldots, s, I d) \in G(r, n): p \mid n s\}| \\
& =\left|\left\{(s, \ldots, s, I d) \in G(r, n): p^{\prime} \mid s\right\}\right| \\
& =\frac{r}{p^{\prime}} .
\end{aligned}
$$

The last equality is a routine computation using the Principle of Inclusion-Exclusion and the first part.

Corollary 4.2 makes it natural to try to characterize the groups $G(r, p, n)$ for which the more refined identity, without intersecting with $[n-1]$, holds.

Corollary 4.3. Let $r, p, n \in \mathbb{P}$, with $p \mid r$. The following are equivalent:

- there is no $q \in[n] \backslash\{1\}$ such that $q \mid p$,

- for all $I \subseteq[n]$,

$$
|\{g \in G(r, p, n): F(g)=I\}|=|\{g \in G(r, p, n): A A(g)=I\}| .
$$

In this case,

$$
\begin{aligned}
& |\{g \in G(r, p, n):|F(g)|=m\}|=|\{g \in G(r, p, n):|A A(g)|=m\}| \\
& =\frac{n !}{p} \sum_{k=m}^{n}(-1)^{k-m} \frac{r^{n-k}}{m !(k-m) !}
\end{aligned}
$$

for all $0 \leq m \leq n$.

Proof. Suppose that there is no $q \in[n] \backslash\{1\}$ such that $q \mid p$. If $I \subseteq[n]$, then $\lambda(I, \emptyset)=\left(1^{n-|I|}\right)$, and so Theorem 4.1 implies

$$
\left|\mathcal{G}_{I, \emptyset}(r, p, n)\right|=(n-|I|) ! \frac{r^{n-|I|}}{p},
$$

as in the previous corollary. On the other hand. If $n \notin I$, then as in the proof of Corollary 4.2 we obtain

$$
\left|\mathcal{G}_{\emptyset, I}(r, p, n)\right|=\operatorname{gcd}\left(\lambda_{1}, \ldots, \lambda_{n-|I|}, p\right)(n-|I|) ! \frac{r^{n-|I|}}{p}=(n-|I|) ! \frac{r^{n-|I|}}{p}
$$

since if $q \in \mathbb{P}$ is such that $q \mid p$ and $q \mid \lambda_{i}$ for $i=1, \ldots, n-|I|$ then $q \leq n$ so, by our hypothesis, $q=1$. If $n \in I$ then $\alpha(\{n\}, I \backslash\{n\})=\left(\alpha_{1}\right)$ for some $\alpha_{1} \leq n-1$ and so, as above

$$
\left|\mathcal{G}_{\{n\}, I \backslash\{n\}}(r, p, n)\right|=\operatorname{gcd}\left(\lambda_{1}, \ldots, \lambda_{n-|I|}, p\right)(n-|I|) ! \frac{r^{n-|I|}}{p}=(n-|I|) ! \frac{r^{n-|I|}}{p},
$$


by Theorem 4.1 and our hypothesis. Therefore

$$
|\{g \in G(r, p, n): F(g) \supseteq I\}|=|\{g \in G(r, p, n): A A(g) \supseteq I\}| .
$$

Applying the Principle of Inclusion-Exclusion to all $I \subseteq[n]$ yields the claimed identity.

Conversely, suppose that there is $q \in[n] \backslash\{1\}$ such that $q \mid p$. If $I=[n] \backslash\{q\}$, then

$$
\begin{aligned}
\mid\{g & \in G(r, p, n): F(g)=I\}|=|\{(\underbrace{0, \ldots, 0, s}_{q}, 0, \ldots, 0, I d) \in G(r, n): p \mid s, s \neq 0\} \mid \\
& =\frac{r}{p}-1 .
\end{aligned}
$$

On the other hand, let $g=\left(s_{1}, \ldots, s_{n}, \sigma\right) \in G(r, p, n)$ be such that $A A(g)=I$. Then we have

- $\left(\sigma(i+1), s_{i+1}\right)=\left(\sigma(i)+1, s_{i}\right)$, for all $i \in[q-1]$,

- $\left(\sigma(i), s_{i}\right)=(i, 0)$, for all $i \in[q+1, n]$.

These two conditions force $\left(s_{1}, \ldots, s_{n}, \sigma\right)=(\underbrace{s, \ldots, s}_{q}, 0, \ldots, 0, I d)$; moreover, we have the conditions $s \neq 0$ and $p \mid q s$. Hence

$$
|\{g \in G(r, p, n): A A(g)=I\}|=\frac{r q}{p}-1
$$

and $|\{g \in G(r, p, n): F(g)=I\}| \neq\{g \in G(r, p, n): A A(g)=I\} \mid$.

The last equality follows as in the proof of Corollary 4.2.

Note that the bijection $\left[\sigma(1)^{s_{1}}, \ldots, \sigma(n)^{s_{n}}\right] \mapsto\left[(n+1-\sigma(n))^{s_{n}}, \ldots,(n+1-\sigma(1))^{s_{1}}\right]$ shows that

$$
\begin{aligned}
& \mid\left\{\left(s_{1}, \ldots, s_{n}, \sigma\right) \in G(r, n):\left(\sigma(i-1), s_{i-1}\right)=\left(\sigma(i)-1, s_{i}\right) \text { if and only if } i \in I\right\} \mid \\
& \quad=|\{g \in G(r, n): A A(g)=n+1-I\}|
\end{aligned}
$$

for all $I \subseteq[n]$ (recall our convention that $\sigma(0)=0$ and $s_{0}=0$, for all $\left(s_{1}, \ldots, s_{n}, \sigma\right) \in$ $G(r, n))$. But, for $J \subseteq[n]$, the cardinality $|\{g \in G(r, n): A A(g)=J\}|$ only depends on $|J|$, by the proof of Corollary 4.3. Thus Corollary 4.3 can also be stated with $\underline{A A}(g)$ in place of $A A(g)$, where

$$
\underline{A A}(g):=\left\{i \in[n]:\left(\sigma(i-1), s_{i-1}\right)=\left(\sigma(i)-1, s_{i}\right)\right\}
$$

for all $g=\left(s_{1}, \ldots, s_{n}, \sigma\right) \in G(r, n)$.

The previous results naturally suggest the following problem. 


\section{Problem 4.4. Compute}

$$
|\{g \in G(r, p, n): F(g)=A A(g)=\emptyset\}|
$$

for all $r, p, n \in \mathbb{P}$ such that $p \mid r$.

In particular, it would be interesting to know if

$$
\lim _{n \rightarrow \infty} \frac{|\{g \in G(r, p, n): F(g)=A A(g)=\emptyset\}|}{r^{n} n !}
$$

exists.

If we think of a permutation of $[n]$ as the result of shuffling a deck of $n$ cards (as Diaconis, Evans, and Graham do in [5]), and of a signed permutation (i.e., an element of the hyperoctahedral group $B_{n}$ ) as the result of shuffling a deck of $n$ cards and then turning some of them face up, then our notion of "adjacent ascent" is not the most natural one. Indeed, an "unseparated pair" in such a "signed" shuffle should be a pair of cards that are adjacent (and hence both face down) at the beginning of the shuffle (say 1 on top and 2 beneath 1 ) and remain in the same relative position at the end of it. So, at the end of the signed shuffle, these two cards should be still adjacent and either both face down (so 1 on the top) or both face up (so 1 on the bottom). Thus we should analyse the integers $i \in[n]$ such that $\sigma(i+1)=\sigma(i)+1$.

It turns out that this set statistic is equidistributed with the one that we consider in this section $(A A(\sigma))$, as we now show. In the following results, we use the usual notation for the classical Weyl groups, as recalled in $\S 2$.

Lemma 4.5. Let $n \in \mathbb{P}$. Then

$$
\begin{aligned}
& \left|\left\{\sigma \in B_{n}:\{i \in[n-1]:|\sigma(i+1)|=|\sigma(i)|+1, \sigma(i) \sigma(i+1)>0\}=I\right\}\right|= \\
& \left|\left\{\sigma \in B_{n}:\{i \in[n-1]: \sigma(i+1)=\sigma(i)+1\}=I\right\}\right|
\end{aligned}
$$

for all $I \subseteq[n-1]$.

Proof. We prove this by exhibiting an appropriate bijection. Let $\sigma \in B_{n}$. Let $[\alpha, \omega] \subseteq[n]$ be such that $\sigma(\alpha-1)>0, \sigma(\omega+1)>0$ and $\sigma(j)<0$ for all $\alpha \leq j \leq \omega$, where the first two conditions are empty if $\alpha=1$ or if $\omega=n$. Let $\hat{\sigma}$ be the element of $B_{n}$ whose window notation is obtained from that of $\sigma$ by changing 
$[\sigma(\alpha), \sigma(\alpha+1), \ldots, \sigma(\omega)]$ to $[\sigma(\omega), \sigma(\omega-1), \ldots, \sigma(\alpha)]$, for all such intervals $[\alpha, \omega]$. The map $\sigma \mapsto \hat{\sigma}$ is an involution of $B_{n}$, and

$$
\left\{i \in[n]: \sigma^{-1}(i+1)=\sigma^{-1}(i)+1\right\}=A A\left((\hat{\sigma})^{-1}\right)
$$

for all $\sigma \in B_{n}$.

Note that the previous result also holds with $D_{n}$ in place of $B_{n}$ since the bijection $\sigma \mapsto \hat{\sigma}$ restricts to $D_{n}$.

Corollary 4.6. Let $n \in \mathbb{P}$. If $n$ is odd, then

$$
\left|\left\{\sigma \in D_{n}: F(\sigma) \cap[n-1]=I\right\}\right|=\left|\left\{\sigma \in D_{n}:\{i \in[n-1]: \sigma(i+1)=\sigma(i)+1\}=I\right\}\right|
$$

for all $I \subseteq[n-1]$

Proof. This follows from Corollary 4.2 and the comment following Lemma 4.5.

Corollary 4.7. Let $n \in \mathbb{P}$. Then

$$
\left|\left\{\sigma \in B_{n}: F(\sigma)=I\right\}\right|=\mid\left\{\sigma \in B_{n}: \sigma(i+1)=\sigma(i)+1 \text { if and only if } i \in I\right\} \mid
$$

for all $I \subseteq[n]$ (with the convention that $\sigma(n+1)=n+1$ for all $\sigma \in B_{n}$ ).

Proof. This follows from Corollary 4.3 and Lemma 4.5.

Corollary 4.8. Let $n \in \mathbb{P}$. Then

$$
\left|\left\{\sigma \in S_{n}: F(\sigma)=I\right\}\right|=\left|\left\{\sigma \in S_{n}: A A(\sigma)=I\right\}\right|
$$

for all $I \subseteq[n]$.

Note that Corollary 4.8 refines Theorem 2.2. Indeed, if $I \subseteq[n-1]$, then

$$
\begin{aligned}
& \left|\left\{\sigma \in S_{n}: F(\sigma) \cap[n-1]=I\right\}\right|= \\
& \left|\left\{\sigma \in S_{n}: F(\sigma)=I\right\}\right|+\left|\left\{\sigma \in S_{n}: F(\sigma)=I \cup\{n\}\right\}\right|
\end{aligned}
$$

and similarly

$$
\begin{aligned}
& \left|\left\{\sigma \in S_{n}: A A(\sigma) \cap[n-1]=I\right\}\right|= \\
& \left|\left\{\sigma \in S_{n}: A A(\sigma)=I\right\}\right|+\left|\left\{\sigma \in S_{n}: A A(\sigma)=I \cup\{n\}\right\}\right|
\end{aligned}
$$

and these two pairs of numbers are equal by Corollary 4.8 . 


\section{Variations}

While there is a well established notion of fixed point of a colored permutation, we feel that for the definition of "adjacent ascent" other possibilities also come fairly naturally to mind. In this section, we study two other such possibilities.

Given $g=\left(s_{1}, \ldots, s_{n}, \sigma\right) \in G(r, n)$, we let

$$
\begin{gathered}
A A^{*}(g):=\left\{i \in[n]:\left(\sigma(i+1), s_{i+1}\right)=(\sigma(i)+1,0)\right\} \\
A A_{*}(g):=\left\{i \in[n]:\left(\sigma(i+1), s_{i}\right)=(\sigma(i)+1,0)\right\} .
\end{gathered}
$$

Theorem 5.1. Let $p, r, n \in \mathbb{P}$ with $p \mid r, I \subseteq[n]$, and $J \subseteq[n-1]$. If $I$ is $J$ compatible, then

$$
\left|\left\{g \in G(r, p, n): F(g) \supseteq I, A A^{*}(g) \supseteq J\right\}\right|=(n-|I|-|J|) ! \cdot \frac{r^{n-|I|-|J|+t}}{p}
$$

where $t$ is the number of $I$-fixed blocks of $P_{J}$ of cardinality at least 2.

Proof. For short, we set $\mathcal{C}_{I, J}=\left\{g \in G(r, p, n): F(g) \supseteq I, A A^{*}(g) \supseteq J\right\}$. Let $\left(s_{1}, \ldots, s_{n}, \tau\right) \in G(r, n)$. It follows easily from our definitions that $\left(s_{1}, \ldots, s_{n}, \tau\right) \in$ $\mathcal{C}_{I, J}{ }^{-1}$ if and only if $p \mid\left(s_{1}+\cdots+s_{n}\right), \tau(i)=i$ and $s_{i}=0$ for all $i \in I$, and $j$ and $j+1$ are adjacent in this order in the window notation of $\left(s_{1}, \ldots, s_{n}, \tau\right)$ and the color of $j+1$ is 0 for all $j \in J$. Therefore, $\left(s_{1}, \ldots, s_{n}, \tau\right) \in \mathcal{C}_{I, J}{ }^{-1}$ if and only if $p \mid\left(s_{1}+\cdots+s_{n}\right), \tau(i)=i$ and $s_{i}=0$ for all $i \in I$, and $t, t+1, \ldots, j$ are adjacent in this order in the window notation of $\left(s_{1}, \ldots, s_{n}, \tau\right)$ and the color of $t+1, t+2, \ldots, j$ is 0 for all blocks $[t, j]$ of $P_{J}$ (note that this condition is vacously satisfied if $t=j$ ). Hence, $\left(s_{1}, \ldots, s_{n}, \tau\right) \in \mathcal{C}_{I, J}^{-1}$ if and only if $p \mid\left(s_{1}+\cdots+s_{n}\right), t, t+1, \ldots, j$ are adjacent in this order in the window notation of $\left(s_{1}, \ldots, s_{n}, \tau\right)$ and the color of $t+1, t+2, \cdots, j$ is 0 for all blocks $[t, j]$ of $P_{J}$ and furthermore $t, t+1, \ldots, j$ appear in positions $t, t+1, \ldots, j$, respectively, and $j$ has color 0 , for all fixed blocks $[t, j]$. Since $I$ is $J$-compatible, all permutations of the free blocks of $P_{J}$ occur in elements of $\mathcal{C}_{I, J}{ }^{-1}$, and so $\left|\mathcal{C}_{I, J}{ }^{-1}\right|=\frac{r^{m+t}}{p} m$ !, where $m$ is the number of free blocks of $P_{J}$ and $t$ is the number of fixed blocks of $P_{J}$ of size at least 2 . The result follows.

With this choice of "adjacent ascent", the analogue of Theorem 2.2 holds for all colored permutation groups $G(r, p, n)$. 
Corollary 5.2. Let $p, r, n \in \mathbb{P}$, with $p \mid r$. Then

$$
|\{g \in G(r, p, n): F(g) \cap[n-1]=I\}|=\left|\left\{g \in G(r, p, n): A A^{*}(g) \cap[n-1]=I\right\}\right|,
$$

for all $I \subseteq[n-1]$.

Proof. The proof is identical to that of the first implication of Corollary 4.2, noting that, for all $I \subseteq[n-1]$, there are no $\emptyset$-fixed blocks of $P_{I}$, as well as no $I$-fixed blocks of $P_{\emptyset}$ of size at least 2 .

Note that the refined identity in Corollary 4.3 does not hold for $A A^{*}(g)$, even when $p=1$. For example, if $n=4, r=2$, and $I=[3,4]$, then $\{g \in G(2,4): F(g)=\{3,4\}\}=\left\{\left(s_{1}, s_{2}, 0,0,2134\right): s_{1}, s_{2} \in\{0,1\}\right\} \cup\{(1,1,0,0,1234)\}$ while

$$
\begin{aligned}
& \left\{g \in G(2,4): A A^{*}(g)=\{3,4\}\right\} \\
& =\{(s, 1,1,0,1234): s \in\{0,1\}\} \cup\left\{\left(s_{1}, s_{2}, s_{3}, 0,2134\right): s_{1}, s_{2}, s_{3} \in\{0,1\}\right\} .
\end{aligned}
$$

We conclude by investigating our second variation of the concept of adjacent ascent. Although at first sight it may seem to be closely related to the first variation, it is in fact equivalent to the concept of adjacent ascent that we studied in $\S 4$.

Theorem 5.3. Let $p, r, n \in \mathbb{P}$, with $p \mid r$. If $I \subseteq[n]$ and $J \subseteq[n-1]$, with $I \cap J=\emptyset$, then

$\left|\left\{g \in G(r, p, n): F(g) \supseteq I, A A_{*}(g) \supseteq J\right\}\right|=|\{g \in G(r, p, n): F(g) \supseteq I, A A(g) \supseteq J\}|$.

Proof. For short, set $\mathcal{D}_{I, J}=\left\{g \in G(r, p, n): F(g) \supseteq I, A A_{*}(g) \supseteq J\right\}$. We show that $\mathcal{D}_{I, J}$ is in bijection with $\mathcal{G}_{I, J}:=\mathcal{G}_{I, J}(r, p, n)$ (see the definition before Theorem 4.1).

Let $\left(s_{1}, \ldots, s_{n}, \tau\right) \in G(r, n)$. By reasoning as in the proof of Theorem 5.1, one can see that $\left(s_{1}, \ldots, s_{n}, \tau\right) \in \mathcal{D}_{I, J}^{-1}$ if and only if $t, t+1, \ldots, j$ are adjacent in this order in the window notation of $\left(s_{1}, \ldots, s_{n}, \tau\right)$ and $t, t+1, \ldots, j-1$ all have color 0 , for all blocks $[t, j]$ of $P_{J}$, and furthermore $t, t+1, \ldots, j$ appear in positions $t, t+1, \ldots, j$, respectively, and $s_{j}=0$, for all fixed blocks $[t, j]$ of $P_{J}$. Let $g \in \mathcal{G}_{I, J}^{-1}$. Define $\hat{g}$ to be the colored permutation whose window notation is obtained from that of $g$ by changing to 0 the colors of $t, t+1, \ldots, j-1$ for every free block $[t, j]$ of $P_{J}$ (recall that, in this case, $t, t+1, \ldots, j$ all appear adjacent in this order and all with the same color in the window notation of $g$ ). The map $g \mapsto \hat{g}$ is a bijection between $\mathcal{G}_{I, J}^{-1}$ and $\mathcal{D}_{I, J}^{-1}$. 


\section{Two bijections}

In this section, we give bijective proofs of some of the results in this paper. More precisely, we give bijective proofs of Corollary 4.2, for $p=1$, and Corollary 5.2.

Recall that the canonical cycle decomposition of a permutation $\sigma \in S_{n}$ is the cycle decomposition of $\sigma$ such that, in each cycle, the leading element is the least element of the cycle and these leading elements form a decreasing sequence. Let $g=\left(s_{1}, \ldots, s_{n}, \sigma\right) \in G(r, n)$. We define two cycle decompositions of $g$ as follows.

The canonical cycle decomposition of $g$ is obtained from the canonical cycle decomposition of $\sigma$ by coloring the leading element $a$ of each cycle with $s_{\sigma^{-1}(a)}$ (i.e., with the color that $a$ has in the window notation) and an element following $j^{c}$ by $s_{j}+c$, where the sum is taken modulo $r$ (so that the entry following an element $j^{c}$ is $\left.g\left(j^{c}\right)\right)$.

The canonical cycle decomposition colored from 0 of $g$ is obtained from the canonical cycle decomposition of $\sigma$ by coloring the leading element $a$ of each cycle with $s_{\sigma^{-1}(a)}$ and an element following $j^{c}$ with $s_{j}$ (so that each element is colored as in the window notation).

For example, if $g=\left[3^{2}, 2^{0}, 5^{2}, 6^{1}, 1^{2}, 4^{1}\right] \in G(3,6)$, then the canonical cycle decomposition of $g$ is

$$
\left(4^{1}, 6^{2}\right)\left(2^{0}\right)\left(1^{2}, 3^{1}, 5^{0}\right)
$$

and the canonical cycle decomposition colored from 0 of $g$ is

$$
\left(4^{1}, 6^{1}\right)\left(2^{0}\right)\left(1^{2}, 3^{2}, 5^{2}\right)
$$

In the spirit of [5], we define two bijections of $G(r, n)$ to itself. Given $g=$ $\left(s_{1}, \ldots, s_{n}, \sigma\right) \in G(r, n)$, we let $\widehat{g}, \widetilde{g} \in G(r, n)$ be defined as follows: write $g$ in its canonical cycle decomposition (respectively, canonical cycle decomposition colored from 0 ) and remove the parentheses to obtain the window notation of $\widehat{g}$ (respectively, $\widetilde{g})$. Note that, if $g \in G(r, p, n)$, then $\widetilde{g} \in G(r, p, n)$ while possibly $\widehat{g} \in G(r, n) \backslash$ $G(r, p, n)$. We let $\rho:=\left[2^{0}, 3^{0}, \ldots, n^{0}, 1^{0}\right]$.

Theorem 6.1. Let $p, r, n \in \mathbb{P}$, with $p \mid r$.

1. If $g \in G(r, n)$ then

$$
A A\left((\widehat{\rho g})^{-1}\right) \cap[n-1]=F(g) \cap[n-1] .
$$


2. If $g \in G(r, p, n)$ then

$$
A A^{*}\left((\widetilde{\rho g})^{-1}\right) \cap[n-1]=F(g) \cap[n-1] .
$$

Proof. By [5, Theorem 4.1], in both cases we only need to take care of the colors.

(1). Let $g=\left(s_{1}, \ldots, s_{n}, \sigma\right),(\widehat{\rho g})^{-1}=\left(r_{1}, \ldots, r_{n}, \tau\right)$, and $\widehat{\rho g}=\left(c_{1}, \ldots, c_{n}, \tau^{-1}\right)$. Suppose $\sigma(i)=i$, for some $i \in[n-1]$. We know $\tau(i+1)=\tau(i)+1$. We need to show that $r_{i+1}=r_{i}$ holds if and only if $s_{i}=0$ holds. Since the colors of $i$ and $i+1$ in the window notation of $\widehat{\rho g}$ are $c_{\tau(i)}=-r_{i}$ and $c_{\tau(i+1)}=-r_{i+1}$ (where the equalities are modulo $r$ ), we show that $c_{\tau(i+1)}=c_{\tau(i)}$ holds if and only if $s_{i}=0$ holds. By construction, $(i+1)^{c_{\tau(i+1)}}$ is not the leading element in its cycle in the canonical cycle decomposition of $\rho g$, and so it is preceded by $i^{c_{\tau(i)}}$; hence $c_{\tau(i+1)}=s_{i}+c_{\tau(i)}$ by the definition of the coloring of the canonical cycle decomposition, and we are done.

(2). Let $g=\left(s_{1}, \ldots, s_{n}, \sigma\right),(\widetilde{\rho g})^{-1}=\left(r_{1}, \ldots, r_{n}, \tau\right)$, and $\widetilde{\rho g}=\left(c_{1}, \ldots, c_{n}, \tau^{-1}\right)$. Suppose $\sigma(i)=i$, for some $i \in[n-1]$. We know $\tau(i+1)=\tau(i)+1$. We now need to show that $r_{i+1}$ is 0 if and only if $s_{i}$ is 0 . Since, as above, the color of $i+1$ in the window notation of $\widetilde{\rho g}$ is $c_{\tau(i+1)}=-r_{i+1}$, we show that $c_{\tau(i+1)}$ is 0 if and only if $s_{i}$ is 0 . But, as above, $(i+1)^{c_{\tau(i+1)}}$ is not the leading element in its cycle in the decomposition of $\rho g$, and so it is preceded by $i^{c_{\tau(i)}}$; hence $c_{\tau(i+1)}=s_{i}$ by the definition of the coloring of the canonical cycle decomposition colored from 0 , and we are done.

Acknowledgments: We would like to thank the referee for useful comments.

\section{References}

[1] C. A. Athanasiadis, Edgewise subdivisions, local h-polynomials and excedances in the wreath product $\mathbb{Z}_{r}>\mathfrak{S}_{n}$, SIAM J. Discrete Math., 28 (2014), 1479-1492.

[2] C. A. Athanasiadis, A survey of subdivisions and local h-vectors, in The mathematical legacy of Richard P. Stanley, Amer. Math. Soc., Providence, RI, 2016, 39-51.

[3] A. Björner, F. Brenti, Combinatorics of Coxeter Groups, Graduate Texts in Mathematics, 231, Springer-Verlag, New York, 2005. 
[4] C.-O. Chow, T. Mansour, Counting derangements, involutions and unimodal elements in the wreath product $C_{r}>\mathfrak{S}_{n}$, Israel J. Math., 179 (2010), 425-448.

[5] P. Diaconis, S. N. Evans, R. Graham Unseparated pairs and fixed points in random permutations, Adv. in Appl. Math., 61 (2014), 102-124.

[6] J. E. Humphreys, Reflection Groups and Coxeter Groups, Cambridge Studies in Advanced Mathematics, no.29, Cambridge Univ. Press, Cambridge, 1990.

[7] T. Mansour, M. Shattuck, Counting permutations by the number of succcessions within cycles, Discrete Math., 339 (2016), 1368-1376.

[8] J. Reilly, S. Tanny, Counting permutations by successions and other figures, Discrete Math., 32 (1980), 69-76.

[9] D. Roselle, Permutations by number of rises and successions, Proc. Amer. Math. Soc, 19 (1968), 8-16.

[10] R. P. Stanley, Enumerative Combinatorics , vol.1, Second Edition, Cambridge Studies in Advanced Mathematics, no.49, Cambridge Univ. Press, Cambridge, 2012.

[11] R. P. Stanley, Enumerative Combinatorics , vol.2, Cambridge Studies in Advanced Mathematics, no.62, Cambridge Univ. Press, Cambridge, 1999.

[12] S. Tanny, Permutations and successions, J. Combin. Theory (Series A) , 21 (1976), 196-202.

[13] D. Yesilyurt, Solving Linear Diophantine Equations and Linear Congruential Equations , Linnaeus University, 2012. 